\title{
Kernos
}

Revue internationale et pluridisciplinaire de religion grecque antique

$28 \mid 2015$

Varia

\section{Embracing the Immigrant. The Participation of Metics in Athenian Polis Religion ( $5^{\text {th }}-4^{\text {th }}$ century $\mathrm{BC})$}

Romain Guicharrousse

\section{OpenEdition}

Édition électronique

URL : http://journals.openedition.org/kernos/2345

DOI : $10.4000 /$ kernos. 2345

ISSN : 2034-7871

Éditeur

Centre international d'étude de la religion grecque antique

Édition imprimée

Date de publication : 1 octobre 2015

Pagination : 280-282

ISBN : 978-2-87562-055-2

ISSN : 0776-3824

Référence électronique

Romain Guicharrousse, « Embracing the Immigrant. The Participation of Metics in Athenian Polis Religion (5 $5^{\text {th }}-4^{\text {th }}$ century BC) », Kernos [En ligne], 28 | 2015, mis en ligne le 01 octobre 2015, consulté le 24 septembre 2020. URL : http://journals.openedition.org/kernos/2345 ; DOI : https://doi.org/ $10.4000 /$ kernos. 2345

Ce document a été généré automatiquement le 24 septembre 2020.

Kernos 


\title{
Embracing the Immigrant. The Participation of Metics in Athenian Polis Religion $\left(5^{\text {th }}-4^{\text {th }}\right.$ century BC)
}

\author{
Romain Guicharrousse
}

\section{RÉFÉRENCE}

WIJMA Sara M., Embracing the Immigrant. The Participation of Metics in Athenian Polis Religion $\left(5^{\text {th }}-4^{\text {th }}\right.$ century BC), Stuttgart, Franz Steiner Verlag, 2014. 1 vol. 17,5 × 24,5 cm, $197 \mathrm{p}$.

(Historia - Einzelschriften, 233). ISBN : 978-3-515-10642-9.

1 Dans une historiographie encore très dépendante des travaux de $\mathrm{D}$. Whitehead publiés il y a bientôt quarante ans, l'ouvrage de $\mathrm{S}$. Wijma (S.W.) sur l'intégration des métèques dans la sphère religieuse attique offre un contrepoint bienvenu. Prônant une perspective sociale dans la lignée de l'« École de Paris ", S.W. souhaite se départir des approches morale (Clerc, Ferguson) et juridique (Whitehead) des études sur les métèques. Elle ne propose certes pas de redéfinir la place des métèques au sein de la cité dans son ensemble, mais de réévaluer leur position dans le seul domaine des activités religieuses (hiéra) et, au-delà, de revoir la chronologie de la mise en place de la métoikia à l'époque classique.

Dans une introduction qui pose très clairement définitions et problèmes, S.W. revient sur l'importance des cultes dans la construction d'une communauté. Dans la lignée des travaux de J. Blok nuançant la définition de la citoyenneté par Aristote, l'A. souligne que, pour les citoyens, la participation aux rituels était au moins aussi importante que la participation à l'Assemblée. L'ouvrage souhaite montrer que chacun participe aux hiéra à la place que lui assigne la cité, quel que soit son statut, son sexe ou son âge. Après cette évocation des liens unissant politique et religieux, S.W. revient sur la définition de l'immigrant en Attique. Ce rappel salutaire, appuyé par une mention des sources à disposition, est accompagné d'un court renvoi à l'historiographie du sujet. 
Enfin, elle justifie le choix de la période considérée : l'attestation de la métoikia à Athènes. On s'étonnera cependant que ce choix ne soit pas discuté, puisque la plupart des savants ne datent pas sa disparition de la fin du Iv siècle, mais plutôt du milieu ou de la fin du III $^{\mathrm{e}}$ siècle $^{1}$. Les deux derniers chapitres utilisent d'ailleurs de nombreux documents du III ${ }^{\mathrm{e}}$ siècle.

3 La première partie de l'étude (chapitres 1 et 2) est consacrée aux fêtes athéniennes où la présence de métèques est attestée, à commencer par les Panathénées. L'attention est mise sur la participation des métèques à la procession au sein de quatre contingents différents. S.W., en étudiant également les groupes de citoyen(ne)s, montre que les séparations se font certes par le statut, mais aussi par l'âge ou le sexe. La polémique historiographique (la participation des métèques est-elle humiliante ou honorifique ?) semble ainsi stérile: chacun participe à sa place, et il faut avant tout voir dans la présence de tou(te)s une manière d'exprimer la cohésion de la communauté lors de la plus grande fête de la cité. L'A. souligne à juste titre que, derrière une pratique, il ne faut pas forcément voir un programme normatif. Enfin, S.W. estime que l'ouverture des Panathénées aux métèques, qu'elle date des années $480-470$, est le premier moment où un groupe d'immigrés est présenté comme un tout cohérent.

4 La seconde étape se place après la loi de Périclès sur la citoyenneté de 451/0. Trois autres fêtes sont présentées: les Lénéennes, pour lesquelles les métèques peuvent participer à la procession, être choreutes et chorèges; les Dionysies urbaines, où ils participent à la seule procession mais peuvent être poètes, acteurs ou musiciens; et les Héphaisteia, où ils partagent une part du sacrifice - mais crue et non cuite comme les citoyens. Dans chaque cas, les métèques sont invités à participer, mais selon des modalités spécifiques qui rappellent qu'ils ne font pas pleinement partie de la communauté. S'opère ainsi à cette période un double mouvement de fermeture de la cité et de délimitation des communautés concomitant avec la volonté de répondre au désir croissant des métèques, parfois installés à Athènes de longue date, de s'intégrer. Ce second $v^{e}$ siècle est à ce titre fort important car les conditions du statut de métèque n'évoluent plus au IV $\mathrm{V}^{\mathrm{e}}$ siècle, non plus que la participation des étrangers aux hiéra de la cité.

5 Dans ces deux chapitres, de manière aussi claire que pédagogique, est résumé le déroulement de chaque festival, les participants sont détaillés et est rappelée la place de chaque fête au sein des cultes de la cité. Cette mise en contexte constitue un apport très important de S.W.: il replace les pratiques dans lesquelles sont impliqués des étrangers au sein des pratiques cultuelles de l'ensemble de la communauté. Ces deux premiers chapitres, convaincants, constituent une avancée importante dans la compréhension de la place des métèques à Athènes au v $v^{\mathrm{e}}$ siècle.

6 La seconde partie de l'ouvrage propose de varier les échelles de l'analyse, et invite également à considérer le $\mathrm{IV}$ siècle. La présence d'étrangers dans les dèmes (chapitre 3) met en question la cohésion de ces petites communautés, présentées comme des cités en miniature. À cette problématique, les dèmes répondent différemment : les démotes de Skambonidai conceptualisent les étrangers comme un tout pour mieux les contrôler; au Pirée, le dème autorise le partage de tous les hiéra, sauf ceux qui sont réservés aux démotes "originels »- manière de souligner que les frontières ne sont jamais franchies ; les Ikariens autorisent l'exercice de la chorégie pour certains festivals (Ikarion). Ce dernier cas est cependant à nuancer, car l'A. appuie sa démonstration sur un texte restitué (IG $\left.\mathrm{I}^{3} 254\right)$, pour lequel S. Milanezi a proposé récemment ${ }^{2}$ une autre 
interprétation : une discussion de la restitution aurait été indispensable. On touche ici à un problème récurrent de l'ouvrage: malgré une parenté intellectuelle revendiquée avec une «École » française, l'A. ne semble pas avoir lu la bibliographie francophone récente sur les communautés, pourtant fournie ${ }^{3}$. In fine, ce chapitre souligne que les dèmes ont eu des réponses diverses, en fonction des contextes et de la démographie, quant à la présence d'étrangers sur leurs territoires. La variété des noms attribués à ceux qui sont extérieurs aux dèmes résulte de la relative autonomie des communautés à cette échelle locale: le statut de métèque n'opère de manière systématique qu'au niveau de la cité.

7 Enfin, le dernier chapitre est consacré aux orgéons thraces de la déesse Bendis. L'A. décrit l'introduction progressive de Bendis en Attique. Ce culte est confié, par la cité, à des orgéons dont l'A. liste l'intégralité des attestations épigraphiques ( $\left(\mathrm{V}^{\mathrm{e}}-\mathrm{I}^{\mathrm{er}}\right.$ siècle). La longue querelle historiographique, débutée en 1903, est bien résumée : depuis Wilhelm puis Ferguson, les orgéons sont séparés en orgéons « citoyens » et « thraces ». Face aux contradictions internes de ce raisonnement, l'ouvrage propose de ne plus voir deux orgéons séparés mais un seul groupe qui s'occupe du culte pour la cité et pour tous ceux qui souhaitent prier la déesse. La démonstration est convaincante, même si elle laisse parfois circonspect: ainsi S.W. suit Schwenk pour qui les hiéropes sont des magistrats de la cité, mais ne commente pas $\mathrm{IG} \mathrm{II}^{2} 1361$ où épimélètes et hiéropes convoquent ensemble l'assemblée des orgéons. Elle se contente de signaler en note (p.150) que cette clause est problématique: un commentaire aurait été bienvenu. Pourtant, le mérite de l'A. est d'affirmer enfin ce que les chercheurs suggéraient depuis longtemps sans jamais pourtant se départir de l'approche wilhelmienne. C'est, concernant ce dossier, l'apport majeur de l'ouvrage. Si l'hypothèse finale, considérant les orgéons thraces comme phratrères, est peu convaincante (argument uniquement $e$ silentio), le seul fait que la gestion de ce culte soit dévolue à des étrangers suffit à faire des Thraces, à l'échelle d'une association, des membres particuliers de la « communauté d'Athéna » (p. 158), pour reprendre cette belle périphrase.

8 L'ouvrage s'achève par une conclusion claire et pédagogique. L'intérêt de l'ouvrage apparaît, condensé : le statut de métèque, progressivement mis en place à travers des mesures d'ordre religieux, fiscal, juridique, ne joue qu'à certains niveaux et chaque échelle de la cité gère à sa manière la présence d'étrangers en son sein.

L'ouvrage comporte trois annexes (vases présentant des métèques en procession; liste des chorèges connus dans les dèmes; liste des orgéons connus en Attique), puis une bibliographie riche et complète pour le monde anglophone, mais datée pour le monde francophone. Suivent enfin les indices: si l'Index général est bien organisé, on s'étonnera des indices des sources, simplement listées sans renvoi aux pages où elles sont utilisées. Au vu de la richesse de la documentation utilisée, c'est regrettable. De plus, l'index épigraphique n'est pas à jour : les $I G \mathrm{I}^{3}$, parus en 2012, sont venus mettre à jour les IG II'.

10 Malgré les quelques réserves émises plus haut, cette étude, sérieuse et documentée, est à présent un passage obligé pour qui souhaite étudier l'histoire des métèques athéniens à l'époque classique. La perspective historique de construction de la métoikia, mise en regard des évolutions de l'intégration dans les hiéra attiques, emporte l'adhésion. Si l'on aurait aimé que l'ouvrage nous mène plus loin chronologiquement, force est de constater qu'il renouvelle en profondeur l'approche des étrangers résidents: longtemps considérés comme des marginaux, ils sont, en contexte religieux et selon des 
modalités spécifiques, bien intégrés dans une cité où chacun est à sa place. Cet ouvrage sera ainsi riche en suggestions pour qui étudie un autre "groupe" dans l'Athènes classique, car la constante remise en perspective dans l'ensemble plus large de la communauté montre combien il est fécond de ne jamais sortir de leur(s) contexte(s) des hommes et des femmes habitant une cité, c'est-à-dire une communauté d'individus.

\section{NOTES}

1. M. NIKU, The Official Status of the Foreign Residents in Athens, 322-120 B.C., Helsinki, 2007.

2. S. MILANEZI, « Les Icariens et le dème des Icariens ( $\left.I G \mathrm{II}^{2} 1178\right)$ : à propos de l'identité politique d'un dème attique », in J.-C. COUVENHES, S. MILANEZI (éd.), Individus, groupes et politique à Athènes de Solon à Mithridate, Tours, 2007, p. 257.

3. Notamment P. ISMARD, La cité des réseaux. Athènes et ses associations. $V I^{e}-I^{e r}$ siècle av. J.-C., Paris, 2010.

\section{AUTEURS}

\section{ROMAIN GUICHARROUSSE}

Université de Paris 1 - École française d'Athènes 